\title{
Antiferromagnetic phase transition in a nonequilibrium lattice of Rydberg atoms
}

\author{
Tony E. Lee, ${ }^{1}$ H. Häffner, ${ }^{2}$ and M. C. Cross ${ }^{1}$ \\ ${ }^{1}$ Department of Physics, California Institute of Technology, Pasadena, California 91125, USA \\ ${ }^{2}$ Department of Physics, University of California, Berkeley, California 94720, USA \\ (Received 5 April 2011; revised manuscript received 19 July 2011; published 12 September 2011)
}

\begin{abstract}
We study a driven-dissipative system of atoms in the presence of laser excitation to a Rydberg state and spontaneous emission. The atoms interact via the blockade effect, whereby an atom in the Rydberg state shifts the Rydberg level of neighboring atoms. We use mean-field theory to study how the Rydberg population varies in space. As the laser frequency changes, there is a continuous transition between the uniform and antiferromagnetic phases. The nonequilibrium nature also leads to a novel oscillatory phase and bistability between the uniform and antiferromagnetic phases.
\end{abstract}

DOI: 10.1103/PhysRevA.84.031402

PACS number(s): $32.80 . \mathrm{Rm}, 42.50 . \mathrm{Nn}, 42.65 . \mathrm{Pc}$

The behavior of matter far from equilibrium is a fascinating area of study. The presence of driving and dissipation can lead to remarkable phenomena that are not possible in equilibrium. This has motivated much research on nonequilibrium physics in classical systems, such as fluids, chemical reactions, and biological media [1,2]. An interesting question is: what novel phases appear when a quantum system is driven far from equilibrium? Recent cold-atom experiments have studied equilibrium quantum systems in great detail, but they are also a natural setting to study nonequilibrium quantum systems due to the tunability of driving and dissipation [3-8].

In this paper, we study a nonequilibrium many-body quantum system interacting via Rydberg blockade. A Rydberg atom is one whose electron is excited to a high energy level $n$. The van der Waals interaction between two atoms in identical Rydberg levels scales as $n^{11}$, and this leads to a blockade effect for large $n$ : when one atom is excited to the Rydberg state, it prevents nearby atoms from being excited. This is the basis for quantum information processing schemes with Rydberg atoms [9-14] and a variety of novel phenomena [15-22]. In these schemes, spontaneous emission should be minimized, since it destroys quantum information. On the other hand, spontaneous emission as a source of dissipation may lead to interesting physics, and it can actually be tuned by using different Rydberg levels.

We study a lattice of atoms continuously excited to the Rydberg state and spontaneously decaying back to the ground state. Consider the Rydberg population of each atom; that is, the fraction of time it spends in the Rydberg state. What is the spatial distribution of the Rydberg population in steady state? Using mean-field theory, we show that, as the laser frequency is varied, the system undergoes a continuous transition between a phase with spatially uniform population and a phase with higher population on every other atom. We call the latter the antiferromagnetic phase, since a two-level atom is formally equivalent to a spin-1/2 particle (ground and excited states correspond to down and up spins, respectively) [23]. The nonequilibrium nature also leads to an oscillatory phase, in which the Rydberg population oscillates periodically in time, and to bistability between the uniform and antiferromagnetic phases. Simulations of the full quantum model in one dimension (1D), where mean-field theory is least accurate, show that there are short-range antiferromagnetic correlations but not long-range order. Our work can be extended to more general dipolar gases and NMR.

First, we describe the Rydberg interaction [14]. Suppose two atoms are in the same Rydberg state $n l j$. There is a dipoledipole matrix element between $|n l j n l j\rangle$ and nearby energy levels, and this interaction shifts the energy of $|n l j n l j\rangle$ by an amount $V$. When the atoms are separated by a small distance $R$, the dipolar interaction dominates $\left(V \approx-C_{3} / R^{3}\right)$ but, for large distances, the van der Waals interaction dominates $\left(V \approx-C_{6} / R^{6}\right.$ ). For mathematical convenience, we use the van der Waals interaction and an $\left|n s_{1 / 2} n s_{1 / 2}\right\rangle$ state, so that the interaction is short range and isotropic. However, it is straightforward to extend the analysis to long range and anisotropic interactions. The value of $C_{6}$ depends on $n, l, j$ and is tabulated in Refs. [24-26].

Consider a lattice of atoms that is uniformly excited by a laser from the ground state to a Rydberg state. The atoms are assumed to be fixed in space. Since the van der Waals interaction decreases rapidly with distance, we assume nearestneighbor interactions. Let $|g\rangle_{j}$ and $|e\rangle_{j}$ denote the ground and Rydberg states of atom $j$. The Hamiltonian in the interaction picture and rotating-wave approximation is $(\hbar=1)$

$$
\begin{gathered}
H=\sum_{j} H_{j}+V \sum_{\langle j k\rangle}|e\rangle\left\langle\left. e\right|_{j} \otimes \mid e\right\rangle\left\langle\left. e\right|_{k},\right. \\
H_{j}=-\tilde{\Delta}|e\rangle\left\langle\left. e\right|_{j}+\frac{\tilde{\Omega}}{2}\left(|e\rangle\left\langle\left. g\right|_{j}+\mid g\right\rangle\left\langle\left. e\right|_{j}\right) .\right.\right.
\end{gathered}
$$

The second term in Eq. (1) is the Rydberg interaction, and $H_{j}$ is the Hamiltonian for a two-level atom interacting with a laser. $\tilde{\Delta}=\omega_{\ell}-\omega_{o}$ is the detuning between the laser and transition frequencies. $\tilde{\Omega}$ is the Rabi frequency, which depends on the laser intensity.

The lifetime of the Rydberg state is limited by several processes: spontaneous emission, blackbody radiation, and superradiance [14]. We account for spontaneous emission from the Rydberg level using the linewidth $\gamma$. When a Rydberg atom spontaneously decays, it usually goes directly into the ground state or first to a low-lying state [27]; the low-lying states are relatively short lived, so we ignore them. We also ignore blackbody radiation and superradiance, both of which transfer 
atoms in a Rydberg level to nearby levels. Blackbody radiation can be minimized by working at cryogenic temperatures [28], and it is not clear if superradiance is important when the interaction $V$ is large [27,29]. Future treatments could account for them by considering several Rydberg levels instead of just one.

Thus, each atom has two possible states, and the system is equivalent to a dissipative spin model. Previous works have added dissipation to other spin models by coupling each spin to a heat bath; in those works, there is global thermal equilibrium, and the spins are described by an effective partition function [30,31]. However, in quantum optics, dissipation from spontaneous emission leads to a nonequilibrium situation, since the coupling to the heat bath is weak and Markovian [23]. The density matrix for the atoms, $\rho$, is described by a master equation that is local in time:

$$
\begin{gathered}
\dot{\rho}=-i[H, \rho]+\mathcal{L}[\rho], \\
\mathcal{L}[\rho]=\gamma \sum_{j}\left(-\frac{1}{2}\left\{|e\rangle\left\langle\left. e\right|_{j}, \rho\right\}+|g\rangle\left\langle\left. e\right|_{j} \rho \mid e\right\rangle\left\langle\left. g\right|_{j}\right) .\right.\right.
\end{gathered}
$$

The nonequilibrium nature is exhibited in the interplay between unitary and dissipative dynamics [4,5], and we are interested in the properties of the steady-state solution of Eq. (3).

Due to the complexity of the full quantum problem, we use mean-field theory. For equilibrium spin models, mean-field theory is useful for determining the existence of different phases [32]. Its predictions are accurate in high dimensions but not in low dimensions. For the current nonequilibrium case, we use the approach of Refs. [4,5]: factorize the density matrix by site, $\rho=\bigotimes_{j} \rho_{j}$, and work with the reduced density matrices, $\rho_{j}=\operatorname{Tr}_{\neq \mathrm{j}} \rho$. This accounts for onsite quantum fluctuations but not intersite fluctuations: for atom $j$, the interaction $|e\rangle\left\langle\left. e\right|_{j} \otimes \sum_{k} \mid e\right\rangle\left\langle\left. e\right|_{k}\right.$ is replaced with the mean field $|e\rangle\left\langle\left. e\right|_{j} \sum_{k} \rho_{k, e e}\right.$. In high dimensions, this is a good approximation since fluctuations of the neighbors average out.

Then the evolution of each $\rho_{j}$ is given by

$$
\begin{gathered}
\dot{w}_{j}=-2 \tilde{\Omega} \operatorname{Im} q_{j}-\gamma\left(w_{j}+1\right), \\
\dot{q}_{j}=i\left[\tilde{\Delta}-\frac{V}{2} \sum_{\langle j k\rangle}\left(w_{k}+1\right)\right] q_{j}-\frac{\gamma}{2} q_{j}+i \frac{\tilde{\Omega}}{2} w_{j},
\end{gathered}
$$

where we have defined the inversion $w_{j} \equiv \rho_{j, e e}-\rho_{j, g g}$ and off-diagonal element $q_{j} \equiv \rho_{j, e g}$. The Rydberg population $\rho_{j, e e}=\left(w_{j}+1\right) / 2$ is the observable measured in the experiment by measuring the scattering rate of each atom. $w_{j}=-1$ and 1 mean that the atom is in the ground and Rydberg states, respectively. Equations (5) and (6) are the optical Bloch equations, except that the Rydberg interaction introduces nonlinearity: the detuning for an atom is renormalized by the excitation of its neighbors [Fig. 1(a)].

Since the system is dissipative, it will end up at an attracting solution, which can be a fixed point, limit cycle, quasiperiodic orbit, or strange attractor [33]. (We have not observed the latter two.) We want to know the following: for given parameter values, how many steady-state solutions are there and are they stable? A solution is stable or unstable if a perturbation to it (a)

(b)
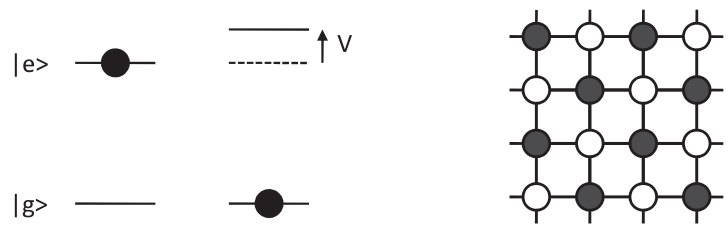

FIG. 1. (a) When one atom is excited to the Rydberg state, it shifts the transition frequency of a neighboring atom by $V$. (b) The lattice is divided into two sublattices.

decays or grows, respectively; the system will end up only in a stable solution.

Equations (5) and (6) always have a steady-state solution, in which the Rydberg population is uniform across the lattice $\left(w_{j}=w, q_{j}=q\right)$. For some parameter values, this uniform solution is stable but, for others, it is unstable to perturbations of wavelength 2 . In the latter case, the lattice divides into two alternating sublattices, and the atoms on one sublattice have a higher Rydberg population than on the other. Hence an antiferromagnetic pattern emerges from the uniform solution through a dynamical instability. To simplify the discussion here, we keep track of only the two sublattices instead of every site [Fig. 1(b)]. We stress that the antiferromagnetic transition is not an artifact of using a bipartite lattice, as shown explicitly in the supplemental material [34].

To simplify the equations, we rescale time by $\gamma$ and also rescale the Rabi frequency $\Omega=\tilde{\Omega} / \gamma$, the detuning $\Delta=\tilde{\Delta} / \gamma$, and the interaction $c=d V / \gamma=-d C_{6} / \gamma R^{6}$, where $d$ is the lattice dimension. Labeling the sublattices 1 and 2, we have

$$
\begin{gathered}
\dot{w}_{1}=-2 \Omega \operatorname{Im} q_{1}-w_{1}-1, \\
\dot{w}_{2}=-2 \Omega \operatorname{Im} q_{2}-w_{2}-1, \\
\dot{q}_{1}=i\left[\Delta-c\left(w_{2}+1\right)\right] q_{1}-\frac{q_{1}}{2}+i \frac{\Omega}{2} w_{1}, \\
\dot{q}_{2}=i\left[\Delta-c\left(w_{1}+1\right)\right] q_{2}-\frac{q_{2}}{2}+i \frac{\Omega}{2} w_{2} .
\end{gathered}
$$

There are six nonlinear differential equations (since $q_{1}$ and $q_{2}$ are complex) and three parameters $(\Omega, \Delta, c)$. The uniform version of these equations $\left(w_{1}=w_{2}, q_{1}=q_{2}\right)$ has been studied before in the context of a medium that interacts with its own electromagnetic field; it is known that there is bistability [35]. We are considering the more general case by letting the sublattices differ.

In the supplemental material [34], we determine the solutions and stabilities for Eqs. (7)-(10). Here, we summarize the main results. Consider first the fixed points; that is, when $\dot{w}_{1}=\dot{w}_{2}=\dot{q}_{1}=\dot{q}_{2}=0$. There are two types of fixed points: the uniform fixed points $\left(w_{1}=w_{2}\right)$ correspond to spatially homogeneous Rydberg excitation, while the nonuniform fixed points $\left(w_{1} \neq w_{2}\right)$ correspond to the antiferromagnetic phase (i.e., when one sublattice has higher excitation than the other).

There are either one or three uniform fixed points, corresponding to the real roots of a cubic polynomial,

$$
\begin{aligned}
f(w)= & c^{2} w^{3}-c(2 \Delta-3 c) w^{2} \\
& +\left[\frac{\Omega^{2}}{2}+\frac{1}{4}+(\Delta-3 c)(\Delta-c)\right] w+(\Delta-c)^{2}+\frac{1}{4} .
\end{aligned}
$$


As the parameters change, pairs of uniform fixed points appear and disappear via saddle-node bifurcations. The uniform fixed points never undergo Hopf bifurcations, so we do not expect limit cycles emerging from them [33].

There are up to two nonuniform fixed points, given by the real roots of a quadratic polynomial,

$$
\begin{aligned}
g(w)= & c^{2}\left(1+4 \Delta^{2}+2 \Omega^{2}\right) w^{2} \\
& -2 c\left[(\Delta-c)\left(1+4 \Delta^{2}\right)+(2 \Delta-c) \Omega^{2}\right] w \\
& +c^{2}\left(1+4 \Delta^{2}\right)-2 c \Delta\left(1+4 \Delta^{2}+2 \Omega^{2}\right) \\
& +\frac{1}{4}\left(1+4 \Delta^{2}+2 \Omega^{2}\right)^{2} .
\end{aligned}
$$

The two roots correspond to $w_{1}$ and $w_{2}$. As the parameters change, the two nonuniform fixed points appear and disappear together.

Since the laser detuning $\Delta$ is the easiest parameter to vary experimentally, we describe what happens as a function of it (Fig. 2). Suppose $\Delta$ starts out large and negative. There is one stable uniform fixed point and no other fixed points. As $\Delta$ increases, the uniform fixed point may undergo a pitchfork bifurcation, in which it becomes unstable and the nonuniform fixed points appear. The bifurcation is supercritical, which means that when the nonuniform fixed points appear, they are stable and coincide with the uniform fixed point [33]. Thus, this is a continuous phase transition between the uniform and antiferromagnetic phases. As $\Delta$ increases further, there is another supercritical pitchfork bifurcation, in which the same uniform fixed point becomes stable again and the nonuniform fixed points disappear. As $\Delta$ increases further toward $\infty$, there is again one stable uniform fixed point and no other fixed points.

Although the nonuniform fixed points are stable when they appear and disappear, they could become unstable in between via a Hopf bifurcation [33]. We find numerically that sometimes the nonuniform fixed points do have Hopf bifurcations [Fig. 2(b)] and give rise to a stable limit cycle, in which $w_{1}$ and $w_{2}$ oscillate periodically in time [Fig. 3(a)]. This oscillatory phase is due to the nonequilibrium nature of the system.
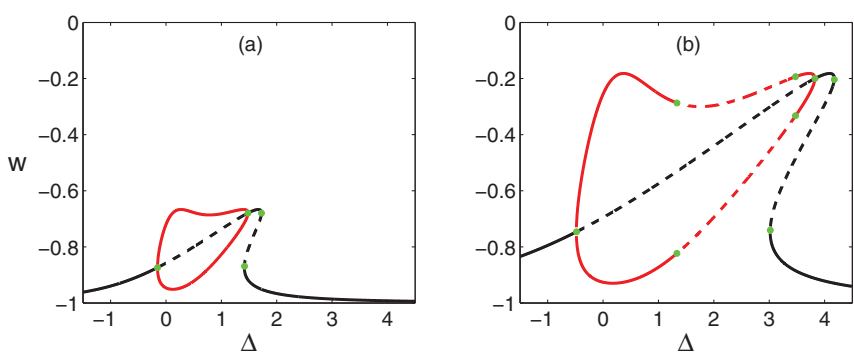

FIG. 2. (Color online) Bifurcation diagram showing fixed-point solutions as function of $\Delta$, with $c=5$ and (a) $\Omega=0.5$ and (b) $\Omega=$ 1.5. The inversion $w$ is -1 (1) when the atom is in the ground (Rydberg) state. Solid (dashed) lines denote stable (unstable) fixed points. Black (grey; red online) lines denote uniform (nonuniform) fixed points. Light grey (green online) points denote bifurcations. In (b), the nonuniform fixed points undergo Hopf bifurcations at $\Delta=3.48$ and 1.33 , and there is a stable limit cycle in that interval [shown in Fig. 3(a)].
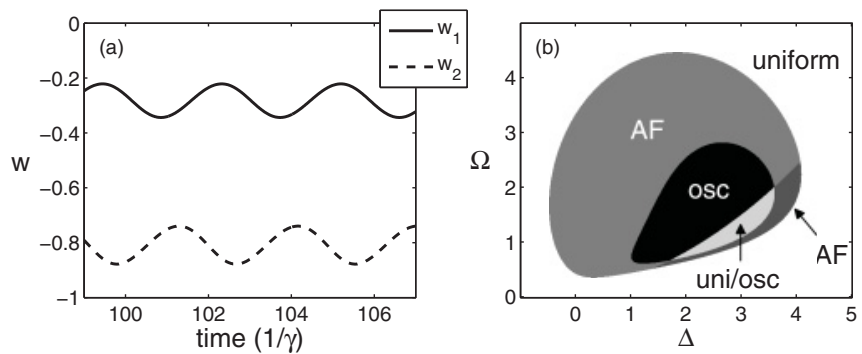

FIG. 3. (a) Oscillatory steady-state solution (limit cycle) for $c=5, \Omega=1.5$, and $\Delta=1.5$. (b) Phase diagram for mean-field theory in $\Omega, \Delta$ space for $c=5$. The system is either in the uniform, antiferromagnetic, or oscillatory phase. It can be bistable between uniform and antiferromagnetic phases or between uniform and oscillatory phases.

Thus, in mean-field theory, there are three phases: uniform, antiferromagnetic, and oscillatory. Figure 3(b) shows a phase diagram in $\Delta, \Omega$ space. For some parameters, the system is bistable between uniform and antiferromagnetic or between uniform and oscillatory [Fig. 2(b)]; the final state depends on the initial conditions.

We also numerically solve the original master equation, Eq. (3), in 1D, where mean-field theory is least accurate. We use fourth-order Runge-Kutta integration to find the steadystate $\rho$ for a chain of length $N=10$. Figure 4(a) shows the correlation as a function of distance, $\left\langle E_{i} E_{i+j}\right\rangle-\left\langle E_{i}\right\rangle\left\langle E_{i+j}\right\rangle$, where $E_{i}=|e\rangle\left\langle\left. e\right|_{i}\right.$. The rapid decay suggests that there is no long range order in $1 \mathrm{D}$, but the fact that it alternates sign means that there is an antiferromagnetic tendency. We also calculate the order parameter, $\left[\left\langle\left(E_{e}-E_{o}\right)^{2}\right\rangle\right]^{1 / 2}$, where the operator $E_{e}=\frac{2}{N} \sum_{i \text { even }} E_{i}$ measures the average Rydberg population on the even sublattice, and $E_{o}$ does likewise for the odd sublattice. The order parameter measures the difference between the two sublattices: it is 0 when they are identical (uniform phase), but positive when they are different (antiferromagnetic and oscillatory phases). The order parameter is largest for roughly the same parameter space, for which mean-field theory predicts the uniform phase to be unstable [compare Fig. 4(b) with Fig. 2(b)]. Thus, mean-field theory captures some qualitative aspects of the full quantum model in $1 \mathrm{D}$, but it remains to be seen whether there is long-range order in higher dimensions, where mean-field
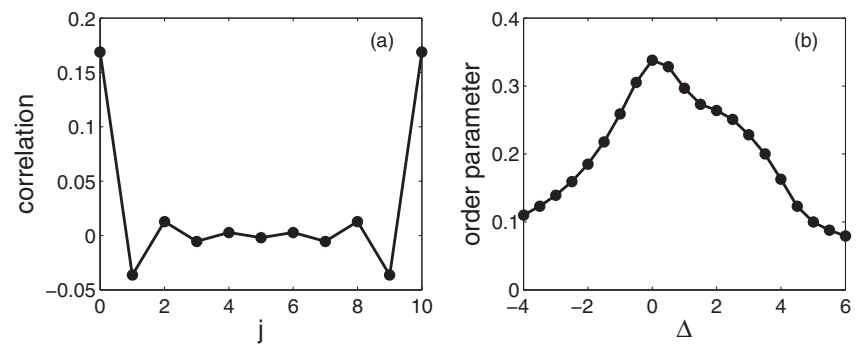

FIG. 4. Numerical solution of master equation for 1D chain of length $N=10$ with periodic boundary conditions. Steady state $\rho$ is found after integrating for time $\gamma t=20$. Parameters are $\Omega=1.5$ and $V=5 \gamma$, which is equivalent to Fig. 2(b). (a) Correlation as a function of distance $j$ for $\Delta=0$. (b) Order parameter as a function of detuning. 
theory is more accurate. Also, the prediction of an oscillatory phase suggests that the emitted light has system-wide temporal correlations; indeed, we have found strong photon correlations, which will be reported in detail elsewhere [36].

Since it is difficult to simulate large systems, experiments with atoms in an optical lattice could provide much information. For example, one can use ${ }^{87} \mathrm{Rb}$ and a two-photon excitation scheme to go from the ground state $5 s_{1 / 2}$ to the Rydberg state $23 s_{1 / 2}$, which has van der Waals interaction $C_{6}=-870 \mathrm{kHz} \mu \mathrm{m}^{6}[25]$ and linewidth $\gamma /(2 \pi)=14.7 \mathrm{kHz}$ at $0 \mathrm{~K}$ [28]. A $d$-dimensional lattice with spacing $R=1.5 \mu \mathrm{m}$ has interaction strength $V=76 \mathrm{kHz}$ and $c=5.2 d$. The Rydberg population of each atom may be measured by imaging the spontaneously emitted photons; in the antiferromagnetic phase, every other atom fluoresces more. Alternatively, the ground-state population may be measured using repeated projective measurements on a $5 s-5 p$ transition. A practical setup would be to use a microscope that both produces the lattice and images the atoms [37].
Thus, a driven-dissipative system of Rydberg atoms has a unique type of antiferromagnetism. The next step is to investigate in more detail how the full quantum model behaves in low dimensions. Our work can be extended to Rydberg states with anisotropic and long-range interactions. Such interactions usually give rise to very rich physics [38], so the nonequilibrium version should be interesting. One can also see what happens when the atoms are not fixed on a lattice but are free to move; this is reminiscent of classical reaction-diffusion systems [1,2]. Finally, we note that a system of interacting Rydberg atoms is similar to a system of spins interacting with each other's magnetic dipolar field $[39,40]$. Thus, when an NMR system is made nonequilibrium with continuous driving and spin relaxation, the spins may form a stable pattern in space.

We thank A. Daley and G. Refael for useful discussions. This work was supported by NSF Grant No. DMR-1003337.
[1] M. C. Cross and P. C. Hohenberg, Rev. Mod. Phys. 65, 851 (1993).

[2] M. C. Cross and H. Greenside, Pattern Formation and Dynamics in Nonequilibrium Systems (Cambridge University Press, Cambridge, 2009).

[3] S. Diehl et al., Nature Phys. 4, 878 (2008).

[4] S. Diehl, A. Tomadin, A. Micheli, R. Fazio, and P. Zoller, Phys. Rev. Lett. 105, 015702 (2010).

[5] A. Tomadin, S. Diehl, and P. Zoller, Phys. Rev. A 83, 013611 (2011).

[6] F. Verstraete et al., Nature Phys. 5, 633 (2009).

[7] J. T. Barreiro et al., Nature (London) 470, 486 (2011).

[8] T. E. Lee and M. C. Cross, Phys. Rev. Lett. 106, 143001 (2011).

[9] D. Jaksch et al., Phys. Rev. Lett. 85, 2208 (2000).

[10] M. D. Lukin et al., Phys. Rev. Lett. 87, 037901 (2001).

[11] L. Isenhower et al., Phys. Rev. Lett. 104, 010503 (2010).

[12] T. Wilk et al., Phys. Rev. Lett. 104, 010502 (2010).

[13] H. Weimer et al., Nature Phys. 6, 382 (2010).

[14] M. Saffman et al., Rev. Mod. Phys. 82, 2313 (2010).

[15] H. Weimer, R. Low, T. Pfau, and H. P. Buchler, Phys. Rev. Lett. 101, 250601 (2008).

[16] J. Honer, H. Weimer, T. Pfau, and H. P. Buchler, Phys. Rev. Lett. 105, 160404 (2010).

[17] G. Pupillo, A. Micheli, M. Boninsegni, I. Lesanovsky, and P. Zoller, Phys. Rev. Lett. 104, 223002 (2010).

[18] I. Lesanovsky, B. Olmos, and J. P. Garrahan, Phys. Rev. Lett. 105, 100603 (2010).

[19] T. Pohl, E. Demler, and M. D. Lukin, Phys. Rev. Lett. 104, 043002 (2010)

[20] J. Schachenmayer et al., New J. Phys. 12, 103044 (2010).

[21] N. Henkel, R. Nath, and T. Pohl, Phys. Rev. Lett. 104, 195302 (2010).
[22] F. Cinti et al., Phys. Rev. Lett. 105, 135301 (2010).

[23] C. Cohen-Tannoudji, J. Dupont-Roc, and G. Grynberg, AtomPhoton Interactions (Wiley, New York, 1992).

[24] K. Singer et al., J. Phys. B 38, S295 (2005).

[25] A. Reinhard, T. C. Liebisch, B. Knuffman, and G. Raithel, Phys. Rev. A 75, 032712 (2007).

[26] T. G. Walker and M. Saffman, Phys. Rev. A 77, 032723 (2008).

[27] J. O. Day, E. Brekke, and T. G. Walker, Phys. Rev. A 77, 052712 (2008).

[28] I. I. Beterov, I. I. Ryabtsev, D. B. Tretyakov, and V. M. Entin, Phys. Rev. A 79, 052504 (2009).

[29] T. Wang et al., Phys. Rev. A 75, 033802 (2007).

[30] P. Werner, K. Volker, M. Troyer, and S. Chakravarty, Phys. Rev. Lett. 94, 047201 (2005).

[31] I. B. Sperstad, E. B. Stiansen, and A. Sudbo, Phys. Rev. B 81, 104302 (2010).

[32] N. W. Ashcroft and N. D. Mermin, Solid State Physics (Brooks/Cole, 1976).

[33] S. H. Strogatz, Nonlinear Dynamics and Chaos (Perseus Books, Cambridge, 1994).

[34] See Supplemental Material at http://link.aps.org/supplemental/ 10.1103/PhysRevA.84.031402 for details on the mean-field steady states.

[35] F. A. Hopf, C. M. Bowden, and W. H. Louisell, Phys. Rev. A 29, 2591 (1984).

[36] T. E. Lee et al. (to be published).

[37] W. S. Bakr et al., Nature (London) 462, 74 (2009).

[38] T. Lahaye et al., Rep. Prog. Phys. 72, 126401 (2009).

[39] J. Jeener, Phys. Rev. Lett. 82, 1772 (1999).

[40] Y.-Y. Lin et al., Science 290, 118 (2000). 